\title{
DIAGNÓSTICO TÉCNICO-ECONÓMICO Y PROPUESTA A NIVEL PREDIAL PARA DOS PRODUCTORES GANADEROS FAMILIARES DEL TERRITORIO NORTE DE SANTA FE, ARGENTINA
}

\author{
Rossler, n. ${ }^{1 ;}$ Zabala, J. M. ${ }^{2}$; Modesto, M. F. ${ }^{3}$; \\ López Cuesta, S. ${ }^{4}$ \& Forni, M. A. ${ }^{5}$
}

\begin{abstract}
RESUMEN
El objetivo del trabajo fue realizar un diagnóstico de dos pequeñas explotaciones ganaderas del norte de la provincia de Santa Fe y proponer y evaluar una estrategia de intervención utilizando la metodología de casos de estudio.

Los sistemas de producción familiares analizados son vulnerables porque los bajos ingresos percibidos ponen en riesgo su existencia al no permitir retribuir la mano de obra familiar y asalariada y el cumplimiento de obligaciones financieras.

La propuesta consiste en incorporar una leguminosa subtropical, doble propósito, que puede incrementar el valor bruto de la producción por la venta de las semillas y por la mejora en la productividad ganadera.
\end{abstract}

Palabras clave: pequeños productores familiares, ganadería familiar, diagnóstico, Macroptilium lathyroides.

\footnotetext{
1.- Grupo de Economía Agraria. Facultad de Ciencias Agrarias. Universidad Nacional del Litoral. Kreder 2805. (3080) Esperanza, Santa Fe, Argentina. Email: nrossler@fca.unl.edu.ar

2.- Programa de Documentación, Conservación y Valoración de la Flora Nativa, Universidad Nacional del Litoral, CONICET. Área de Mejoramiento Genético Vegetal. FCA (UNL).

3.- Cátedra de Investigación de mercados. Facultad de Ciencias Económicas. Universidad Nacional del Litoral. Moreno 2557. S3000 CVE, Santa Fe, Santa Fe, Argentina.

4.- Cátedra de Comercio Exterior. FCE (UNL). Responsable del Programa de Fortalecimiento de Pymes de la Universidad Nacional del Litoral. Bv. Pellegrini 2750, S3000 CVE, Santa Fe, Santa Fe, Argentina.

5.- Cátedra de Mecanización Agrícola. FCA (UNL).

Manuscrito recibido el 26 de marzo de 2020 y aceptado para su publicación el 29 de mayo de 2020.
}

Rossler, N.; Zabala, J.M.; Modesto, M.F.; López Cuesta, S.; Forni, M.A. Diagnóstico técnico-económico y propuesta a nivel predial para dos productores ganaderos familiares del territorio norte de Santa Fe, Argentina. FAVE - Ciencias Agrarias 20 (1): 347-360. CC BY-NC-SA 4.0 


\begin{abstract}
Technical-economic diagnosis and proposal at farm level for two family livestock producers in the northern territory of Santa Fe, Argentina.

The objective of this paper was to make diagnose of two small livestock farms in the north of the province of Santa Fe and propose and evaluate an intervention strategy using the case study methodology.

The analysed family production systems are vulnerable because the low perceived incomes can put their existence at risk by not allowing remuneration for family and salaried labour and the fulfilment of financial obligation.

The proposal at farm level consists of incorporate a subtropical legume, dual purpose, which can increase the production gross value by the sale of seeds and by the improvement in livestock productivity.
\end{abstract}

Key words: small family producers, family livestock, diagnosis, Macroptilium lathyroides.

\section{INTRODUCCIÓN}

La agricultura familiar posee una importancia predominante en la obtención de alimentos (seguridad alimentaria), la generación de empleo agrícola, la mitigación de la pobreza, la conservación de la biodiversidad y de las tradiciones culturales (Salcedo et al., 2014).

En el artículo 1 de la Resolución del Grupo Mercado Común (GMC) N²5/07 se define a la agricultura familiar a partir de tres criterios claves: a) la mano de obra es fundamentalmente familiar (aunque de forma limitada puede existir la figura de contratados), b) es la propia familia la responsable directa de las actividades agropecuarias, la que además reside en la explotación (o en una localidad cercana) y c) los recursos disponibles se adaptan a la capacidad productiva de la familia, las actividades que desarrollan y el nivel tecnológico implementado. El Instituto Interamericano de Cooperación Agrícola (MAG, IICA, 2014), considera a las explotaciones familiares como aquellas en las que el principal aporte de mano de obra proviene de los miembros de la familia, recurriendo ocasionalmente a mano de obra contratada, utilizan una baja proporción de insumos y la producción es destinada a autoconsumo y venta al mercado; además, el desempeño de este tipo de explotaciones está directamente vinculado a los objetivos de la familia propietaria y a la posibilidad de acceder a los recursos productivos. A partir de los criterios mencionados, cada país elabora su propia definición de agricultura familiar: en Argentina, el perfil de los agricultores familiares se define en el artículo 5 de la Ley 27.118 (Ministerio de Justicia y Derechos Humanos. Presidencia de la Nación, 2014)

Según Tommasino y Bruno (2006), la lógica de producción de los agricultores familiares, además de obtener ganancias, es asegurar la sobrevivencia de la unidad de producción que le permite trabajar y mantener sus condiciones de vida. La familia y la unidad de producción actúan como un conjunto. 
Considerando el censo nacional agropecuario del año 2002, aproximadamente el 75\% de los establecimientos agropecuarios en Argentina son de carácter familiar.

Estos sistemas familiares, se caracterizan por poseer una superficie individual inferior a la que poseen las explotaciones no familiares (REAF, 2016) y son, según Azcuy Ameghino (2010), los que más afectados se encuentran por los procesos de concentración del capital en el sector agropecuario, con riesgos de desaparición. De aquí la importancia de la implementación de algunas estrategias que eviten esta situación.

Fernández (2017) expone algunas estrategias llevadas adelante por los pequeños productores que persisten pese a los cambios coyunturales, entre las que destaca la infravaloración de sus propios recursos, la pluriactividad y recurrir a la contratación de servicios como estrategia defensiva, además de considerar los ingresos extraprediales de los integrantes de las familias propietarias como subsidios para sus actividades agropecuarias. La diversificación productiva también es utilizada como una estrategia de mitigación de riesgos productivos y de mercado. Respecto de las estrategias productivas, la ganadería en el norte de Argentina, se basa principalmente en el uso del pastizal natural, con un escaso aporte de tecnología (como, por ejemplo, la siembra de especies forrajeras).

El Programa de Documentación, Conservación y Valoración de la Flora Nativa de la Universidad Nacional del Litoral (PRODOCOVA), trabaja en el mejoramiento de forrajeras nativas para el norte de Argentina, y en la adopción de estas especies por parte de pequeños productores, con un doble propósito: uso del forraje y venta del excedente de semillas. A partir de este programa, y en el marco del proyecto denominado "Elaboración del plan de negocios para la producción de semillas leguminosas nativas con doble propósito forrajero y semillero" ${ }^{1}$, se realizó un diagnóstico técnico económico a dos productores ganaderos familiares del departamento San Javier (provincia de Santa Fe) y se evaluó una alternativa a nivel predial que permita a los productores diversificar su producción y mejorar sus ingresos.

\section{MATERIALES Y MÉTODOS}

Se trabajó con la metodología de Estudio de Casos siguiendo los lineamientos de Apollin y Eberhart (1999). Cada sistema productivo o explotación es un caso de estudio. De acuerdo a estos autores, un sistema de producción se compone de todas las actividades agropecuarias y no agropecuarias combinadas en diversos subsistemas: de cultivo, de crianza, de transformación y actividades no agrícolas. En este trabajo se consideraron como actividades agropecuarias a los tres primeros subsistemas y se describieron los elementos que las constituyen: mano de obra, capital de producción (herramientas y equipos, infraestructura, hacienda) y medio explotado (tierra).

La selección de los 2 casos de estudio se realizó en base a los productores ganaderos familiares que trabajan junto al PRODOCOVA en el desarrollo de la tecnología

1.- Desarrollo de Negocios Tecnológicos 2016. Agencia Santafesina de Ciencia, Tecnología e Innovación ASaCTeI. Ministerio de Ciencia, Tecnología e Innovación Productiva. Gobierno de Santa Fe. Director del proyecto: Marcelo Zabala. 
de producción de forraje y semillas de una forrajera nativa, Macroptilium lathyroides (L.) Urb. Ambos productores familiares (de ahora en más caso 1 y 2) se interesaron en participar de este trabajo. Sus explotaciones se ubican en el departamento San Javier, en la provincia de Santa Fe, y se dedican a la cría de ganado vacuno. De acuerdo a las definiciones de agricultura familiar, estos productores integrarían el segmento de agricultura familiar inserta en los mercados y que genera excedentes (Salcedo et al., 2014).

Se realizaron entrevistas a los propietarios de los establecimientos ganaderos, en la que se recolectaron datos, mediante el uso de un cuestionario semiestructurado, correspondientes a un ciclo productivo. Surgieron algunas dificultades en la recolección de datos, generadas por la falta de registros en las explotaciones.

En sendos casos se realizó un diagnóstico de su situación actual, estimándose resultados económicos (considerando la limitante de ausencia de registros), y se propuso una alternativa de diversificación productiva, para mejorar sus ingresos agropecuarios y mitigar riesgos productivos y de mercado. Los precios que se utilizaron corresponden a marzo de 2020 y provinieron de comerciantes locales y revistas especializadas.

En relación a la estimación de resultados económicos, se considera que la racionalidad en la toma de decisiones de estos productores familiares es diferente a la del empresario capitalista, básicamente, porque tienen objetivos diferentes (cada sistema de producción posee una racionalidad económica específica más allá de la maximización de beneficios, por ejemplo, asegurar la obtención de un ingreso mínimo que le permita a la familia propietaria cumplir sus necesidades básicas y la sobrevivencia de la unidad de producción en el tiempo). Es por esto que no se utilizaron conceptos como, por ejemplo, rentabilidad. Se estimó el valor agregado bruto de la producción (VAB), obtenido a partir de la diferencia entre la producción bruta $(\mathrm{PB}=$ ingresos por venta de productos - consumo familiar +/- diferencia de inventario) y el consumo intermedio (CI, gastos generados por el proceso productivo) medidos en unidades monetarias, haciendo un tratamiento especial del rubro de la mano de obra² (Apollin y Eberhart, 1999), que no se incluyó dentro de los gastos. De acuerdo a Gittinger (1983), la mano de obra familiar no se considera un costo, sino que forma parte del beneficio neto, entonces, si aumentan los beneficios, se incrementa también el ingreso familiar independientemente del salario que se esté obteniendo. De la misma forma se evaluó la estrategia de intervención a nivel predial propuesta.

Una de las alternativas posibles de mejora para los dos sistemas ganaderos estudiados es la intersiembra de especies forrajeras a los pastizales naturales (base alimenticia de los rodeos de esta zona), con el objetivo de incrementar la cantidad y calidad del forraje ofrecido y producir semilla para uso propio y venta del excedente. Por lo tanto, la estrategia propuesta consiste en la incorporación de una leguminosa estival anual, M. lathyroides (cultivar Mancebo) en una proporción entre 1 y 5 hectáreas de cada establecimiento. El cultivar es propiedad de la Universidad Nacional del Litoral (INASE, 2016). La importancia de la inclusión

2.- El trabajo familiar o asalariado, el costo de alquiler de la tierra, el transporte de los productos, etc. no constituyen consumos intermedios. 
de esta leguminosa al planteo de uso del suelo radica en que se encuentra -en escasa proporción- en los pastizales, incrementa la calidad de la dieta animal (permite obtener 2.000 a $4.000 \mathrm{~kg}$ de materia seca por hectárea en un corte, con $60 \%$ de digestibilidad y $20 \%$ de proteína bruta aproximadamente (Dr. Zabala, comunicación personal) y es capaz de fijar nitrógeno atmosférico al suelo mejorando sus niveles de fertilidad. Por otro lado, el cultivo de esta leguminosa, permite mejorar los ingresos de las explotaciones familiares a través de la producción de semillas de forrajeras nativas mejoradas (con un rendimiento probable de entre 100 y $300 \mathrm{~kg} / \mathrm{ha}$ ) posibles de ser comercializadas (diversificación de la producción).

Se trata de una producción artesanal. Si bien la preparación del suelo y la siembra no difieren de otras forrajeras utilizadas en la zona, en el caso de la especie bajo estudio, debe realizarse un corte a las plantas arraigadas porque está comprobado que mejora la producción de semillas. El forraje obtenido mediante este corte está disponible para ser consumido por los animales de la explotación. En relación a la cosecha, estas especies tienen una dificultad, la producción de semillas no se concentra en un momento, sino que se extiende durante aproximadamente un mes. Además, los frutos tienen una característica que es la dehiscencia explosiva (lo que hace que se pierda semilla). A través de trabajos de investigación realizados por el PRODOCOVA se ha determinado el momento en el que la producción de semillas es máxima y la dehiscencia mínima (Zabala et al, 2015). La cosecha se realiza cortando las plantas que contienen los frutos. Este material debe ser trasladado a un tinglado o galpón y ser depositadas, preferentemente, sobre una lona esperando que se produzca la dehiscencia y se desprendan las semillas que se mantienen almacenadas durante algunas semanas. Se ayuda al proceso de secado mediante remoción del material, es decir, día por medio se deben dar vuelta los frutos con horquillas hasta que los mismos se sequen y la mayor parte de la semilla salga por dehiscencia y queden depositadas sobre la lona. Lo que queda en los frutos se pasa por una máquina trilladora. La semilla recolectada se guarda en bolsas para tal fin. De las mismas se extraen muestras que permiten determinar la calidad (pureza, dureza, poder germinativo, entre otros) y, si es adecuada, está lista para ser comercializada.

Para realizar la siembra, el corte del forraje y la cosecha se necesitan maquinarias especiales, que pueden ser obtenidas por los productores de manera cooperativa o asociativa (no más allá de 5 productores por maquinaria para evitar problemas en relación al momento de uso de las mismas). El valor de la sembradora es de aproximadamente \$512.741 y el de la cosechadora de $\$ 1.648 .095$ (precios correspondientes al mes de marzo de 2020).

La producción de estas semillas es innovadora y existen firmas comerciales zonales interesadas en este negocio. Respecto de la comercialización, sería importante lograr la venta a granel por parte de los productores a firmas comerciales que se ocupen de la limpieza y acondicionamiento de la semilla y los trámites ante el INASE para la comercialización.

En la estimación del PB generado por la venta de las semillas de $M$. lathyroides se consideró el precio de la semilla de Melilotus sp. (costo de oportunidad externo por no existir un valor de mercado). 


\section{RESULTADOS Y DISCUSIÓN}

\section{Descripción de los casos de estudio}

Las explotaciones en las que se trabajó se ubican en el departamento San Javier, en la Zona Homogénea Vera, subzona ganadera del bajo de los saladillos, de acuerdo a la caracterización realizada por Castignani (2011) en función de las condiciones económicas, sociales y ambientales que condicionan el desarrollo de determinados sistemas productivos. El departamento San Javier es predominantemente ganadero, siendo la cría la actividad principal (Santangelo y Gil, 2016).

En relación al lugar de residencia, el productor 1 vive en la misma explotación en una casa que cuenta con un dormitorio, un baño en el interior, techo de paja y chapa y piso de cemento; además mencionó que cuenta con luz eléctrica. Este productor tiene además una casa en la localidad de Alejandra (departamento San Javier). El productor 2, no aportó detalles al respecto, aunque su lugar de residencia no es en la explotación, sino en la localidad de Alejandra.

Medio explotado: tierra. Los productores familiares 1 y 2 poseen, respectivamente, 48 ha y15 ha propias, cuyo valor en el departamento San Javier oscila entre 1.200 y 2.400 dólares por hectárea. En ambos casos, la superficie se encuentra muy por debajo del área promedio (259 ha) de las explotaciones agropecuarias declaradas en el departamento San Javier en las encuestas ganaderas (IPEC, 2016). El acceso a la tierra es por sucesión en el caso 1 y por herencia y compra en el caso 2. Ambas familias llevan años en la actividad: 47 años en el caso 1 y 31 años en el caso 2.

Los establecimientos no se encuentran ubicados en zona de islas, lo que es una ventaja por las características propias de ese tipo de ganadería. No obstante, el productor 2 mencionó que tuvo que retirar 3 veces la hacienda del campo en los últimos 5 años debido a crecientes del río.

Capital de producción (hacienda, herramientas y equipos,

infraestructura)

\section{* Hacienda}

En la tabla 1, se muestra la composición del rodeo existente en los establecimientos.

Los animales criados son cruza. Los rodeos de estos productores son pequeños en relación al promedio del departamento (según las encuestas ganaderas). Esta menor cantidad de animales se vincula directamente a la cantidad de recursos existentes (tierra y mano de obra) y a la imposibilidad de crecer (aumentar el tamaño -magnitud- o el valor agregado, reflejado en el aumento

Tabla 1. Existencia ganadera.

Table 1: Herd size.

\begin{tabular}{|l|l|c|c|c|c|c|c|c|}
\hline & & Vacas & $\begin{array}{c}\text { Vaq. } \\
\text { preñadas }\end{array}$ & $\begin{array}{c}\text { Vaq. de } \\
2 \text { a 3 años }\end{array}$ & $\begin{array}{c}\text { Vaq. de } \\
1 \text { a 2 años }\end{array}$ & Terneros & Terneras & Toros \\
\hline \multirow{2}{*}{ Caso 1 } & $\mathrm{cab}$ & 50 & 11 & 7 & 5 & 13 & 17 & 1 \\
\cline { 2 - 9 } & $\mathrm{kg} / \mathrm{cab}$ & 400 & 330 & 260 & 230 & 200 & 200 & 600 \\
\hline \multirow{2}{*}{ Caso 2} & $\mathrm{cab}$ & 20 & 0 & 4 & 4 & 5 & 6 & 1 \\
\cline { 2 - 9 } & $\mathrm{kg} / \mathrm{cab}$ & 390 & 0 & 320 & 220 & 140 & 140 & 450 \\
\hline
\end{tabular}

Elaboración propia 
del patrimonio neto a través del tiempo) de este tipo de explotaciones de acuerdo a sus resultados económicos.

* Herramientas y equipos

El valor del capital fijo inanimado total es bajo. Ambos casos disponen de herramientas manuales para desarrollar actividades en la explotación, entre lo que mencionaron: recados, arneses, palas, machetes, motosierras, motobombas, boyeros, tenazas, señaladoras, pinzas para caravanas y marcas. El productor 1 cuenta con un tractor obsoleto.

$$
\text { * Infraestructura }
$$

El productor 1 posee un galpón abierto de chapa y postes de madera de $28 \mathrm{~m}^{2}$, en tanto que el productor 2 posee un depósito de herramientas.
Mano de obra. En la tabla 2 se muestra el aporte de mano de obra familiar en las explotaciones en estudio.

El productor 1 vive en el campo. Se trata de una mujer viuda, con tres hijas. Según declaró, trabaja en la explotación, con una dedicación de 56 horas semanales. Las hijas trabajan fuera de la explotación, por decisión propia. Sin embargo, de manera informal, han comprado vacas que se incorporaron al rodeo de cría en el campo. Contrata un empleado rural de 34 años, quien dedica 20 horas semanales a las tareas operativas. La mujer mencionó que la demanda laboral es constante durante todo el año. Además del empleado permanente, contrata 1 empleado eventual si es necesario. Siguiendo los conceptos de Azcuy Ameghino (2010), este

Tabla 2. Composición de la familia y aportes de mano de obra al sistema productivo. Table 2: Family composition and labor contributions to the productive system.

\begin{tabular}{|c|c|c|c|c|c|}
\hline Caso & $\begin{array}{l}\text { Miembro de } \\
\text { la familia }\end{array}$ & $\begin{array}{l}\text { Edad } \\
\text { (años) }\end{array}$ & $\begin{array}{l}\text { Nivel de } \\
\text { estudio }\end{array}$ & $\begin{array}{l}\text { Actividades en la } \\
\text { explotación }\end{array}$ & $\begin{array}{c}\text { ¿Cobran un salario por } \\
\text { trabajo fuera de la } \\
\text { explotación? }\end{array}$ \\
\hline \multirow{4}{*}{1} & Mujer & 69 & Terciario & $\begin{array}{l}\text { Administración } \\
\text { tiempo completo }\end{array}$ & Jubilación y pensión \\
\hline & Hijo 1 & 25 & Universitario & No & $\mathrm{Si}$ \\
\hline & Hijo 2 & 27 & Secundario & No & $\mathrm{Si}$ \\
\hline & Hijo 3 & 34 & Secundario & No & $\mathrm{Si}$ \\
\hline \multirow{6}{*}{2} & Esposo & 55 & Primario & $\begin{array}{l}\text { Administración y } \\
\text { tareas generales }\end{array}$ & Si \\
\hline & Esposa & 50 & Primario & No & Si \\
\hline & Hijo 1 & 26 & Secundario & Tareas generales & Si \\
\hline & Hijo 2 & 25 & Terciario & No & $\mathrm{Si}$ \\
\hline & Hija 3 & 21 & Secundario & No & No \\
\hline & Hijo 4 & 18 & Primario & Tareas generales & Si \\
\hline
\end{tabular}

Elaboración propia 
caso, se trataría de una explotación de "base familiar", considerando la contratación de mano de obra remunerada permanente.

En el caso 2, la familia está constituida por un matrimonio y cuatro hijos. Ellos no viven en el campo. El padre de familia destina 32 horas semanales a las tareas dentro de la explotación, pero además trabaja fuera con una dedicación de 30 horas por semana. Dos de los cuatro hijos destinan, respectivamente, 32 y 6 horas por semana a realizar tareas generales en la explotación, pero también trabajan fuera con una dedicación de 10 y 44 horas cada uno. La madre trabaja fuera del campo, con una dedicación de 30 horas semanales y, el hijo de 25 años, es docente y tampoco trabaja en el campo. El dueño de la explotación mencionó que suelen tener mayor demanda laboral en los meses de marzo y octubre, debido a las campañas de vacunación antiaftosa. No contratan empleados adicionales, por lo que se trata de una explotación familiar.

Se observa también en la tabla 2 , que algunos miembros de las familias reciben ingresos extraprediales, bajo las formas de pensiones, jubilaciones y salarios. Estos productores, en función de los recursos con los que cuentan, se ubicarían dentro del estrato de pequeño productor familiar, de acuerdo a una caracterización realizada por Obschatko (2007). La autora describe a este tipo como productores que no disponen de la suficiente capacidad productiva como para vivir exclusivamente de la actividad agropecuaria y se ven obligados a tener ingresos extraprediales.

\section{Análisis de la actividad ganadera}

Los productores realizan cría vacuna sobre pastizales naturales, sin participación de pasturas cultivadas. Ambos suplementan escasamente con balanceados. El productor 1 utiliza, además, batatas como suplementación. Estos sistemas son característicos de la zona.

Parámetros técnicos de la actividad. En la Tabla 3 se muestran los parámetros del rodeo vacuno para cada caso de estudio. Es necesario recordar, en este punto, la escasa o nula existencia de registros en los establecimientos, por lo que algunos parámetros se estimaron a partir de datos existentes y otros fueron mencionados por los productores en las respectivas entrevistas.

Los productores entrevistados no realizaron descartes de vaquillas de 1 a 2 años en el ciclo analizado.

En la tabla se observan buenos indicadores de destete, una carga animal elevada para la zona y buena edad de primer entore de las vaquillonas. No se evaluó el estado corporal de los animales al momento de las entrevistas. El productor 2, tuvo una elevada mortandad de jóvenes en el ciclo analizado.

Tabla 3. Parámetros del rodeo.

Table 3: General herd parameters.

\begin{tabular}{|l|c|c|c|c|c|c|c|c|}
\hline & $\begin{array}{c}\text { Carga de } \\
\text { vientres } \\
\text { (cab/ha) }\end{array}$ & $\begin{array}{c}\text { Destete } \\
(\%)\end{array}$ & $\begin{array}{c}\text { Descarte } \\
\text { de } \\
\text { vientres } \\
(\%)\end{array}$ & $\begin{array}{c}\text { Descarte } \\
\text { de } \\
\text { vaquillas } \\
\text { de 2 a 3 } \\
\text { años (\%) }\end{array}$ & $\begin{array}{c}\text { Vida útil de } \\
\text { los toros } \\
\text { (años) }\end{array}$ & $\begin{array}{c}\text { Edad de } \\
\text { entore de } \\
\text { vaquillonas } \\
\text { (meses) }\end{array}$ & $\begin{array}{c}\text { Porcentaje } \\
\text { de } \\
\text { mortandad } \\
\text { de adultos } \\
(\%)\end{array}$ & $\begin{array}{c}\text { Porcentaje } \\
\text { de } \\
\text { mortandad } \\
\text { de jóvenes } \\
(\%)\end{array}$ \\
\hline Caso 1 & 1,27 & 87 & 2 & 0 & 4 & 15 & 1,4 & 2,4 \\
\hline Caso 2 & 1,33 & 79 & 9 & 11 & 2 & 15 & 4,55 & 12 \\
\hline
\end{tabular}

Fuente. Elaboración propia en base a datos relevados mediante encuestas. 
En la tabla 4 se muestra la venta de carne del ciclo productivo. El productor 1 vendió a acopiadores principalmente, en tanto que el productor 2 lo hizo directamente en remates feria. Los principales meses de venta de terneros fueron marzo y abril. El productor 2, realizó los descartes de vacas en noviembre.

La importancia de la actividad ganadera radica en que funciona como una caja de ahorro; le da la posibilidad al productor de vender los animales y generar liquidez en los momentos en que lo necesitan.
Estimación de resultados económicos. En la tabla 5, se muestra el valor agregado bruto de cada caso. El CI (gastos) está conformado por los rubros de sanidad del rodeo y alimentación (suplementación). En el año de análisis, los productores no compraron hacienda. Tampoco se consumieron los animales de producción propia.

Tabla 4. Venta de carne.

Table 4: Sale of meat.

\begin{tabular}{|l|c|c|c|c|c|c|}
\hline \multirow{2}{*}{ Categoría de venta } & \multicolumn{3}{|c|}{ Caso 1 } & \multicolumn{3}{c|}{ Caso 2 } \\
\cline { 2 - 7 } & Cab & $\mathrm{kg} / \mathrm{cab}$ & $\mathrm{kg}$ totales & Cab & $\mathrm{kg} / \mathrm{cab}$ & $\mathrm{kg}$ totales \\
\hline Terneros & 7 & 160 & 1.120 & 4 & 190 & 760 \\
\hline Terneras & 2 & 160 & 320 & 0 & 0 & 0 \\
\hline Vacas de rechazo & 1 & 420 & 420 & 2 & 390 & 780 \\
\hline $\begin{array}{l}\text { Vaquillas de 2 a 3 años } \\
\text { de descarte }\end{array}$ & 0 & 0 & 0 & 1 & 330 & 330 \\
\hline Total & & & 1.860 & & & 1.870 \\
\hline Total / hectárea & & & 38,75 & & & 124,67 \\
\hline
\end{tabular}

Elaboración propia

Tabla 5. Valor agregado bruto de las explotaciones.

Table 5: Gross value added in farms.

\begin{tabular}{|l|c|c|c|c|c|}
\hline & $\mathrm{PB}(\$ / a n ̃ o)$ & $\mathrm{Cl}$ (\$/año) & $\mathrm{VAB}(\$ / a n ̃ o)$ & $\mathrm{VAB}(\$ / \mathrm{ha})$ & $\mathrm{VAB}\left(\$ / \mathrm{EH}^{3}\right)$ \\
\hline Caso 1 & 118.659 & 59.025 & 59.634 & 1.242 & 41.996 \\
\hline Caso 2 & 50.301 & 20.256 & 30.045 & 2.003 & 20.649 \\
\hline
\end{tabular}

Elaboración propia

3.- EH. Equivalentes hombre estimados en función de la edad de los trabajadores y las horas semanales dedicadas a trabajar en la explotación. Un equivalente hombre es igual a un año de ocupación plena (300 jornadas) de un hombre en edad de trabajar. 
El VAB obtenido es un indicador de la riqueza generada en los sistemas productivos por el trabajo familiar, en el caso del productor 2, y familiar más asalariado en el caso del productor $1^{4}$. Este resultado, luego, debe destinarse al pago del arrendamiento de la tierra, en caso de que exista, de intereses de préstamos obtenidos, de impuestos, servicios y mano de obra asalariada. Finalmente, el sobrante denominado ingreso neto por Apollin y Eberhart (1999), se destina a remunerar la mano de obra familiar. Esta remuneración al trabajo familiar es, según los autores mencionados, la riqueza de la que disponen los trabajadores familiares para vivir durante el año y crecer (aumentar el patrimonio neto).

En la tabla 5 se puede ver que el caso 2 es más eficiente en el uso de la tierra, en tanto que la productividad del trabajo (la riqueza producida por cada trabajador) es mayor en el caso 1.

El VAB logrado en los casos 1 y 2 equivale respectivamente a 4,40 y 2,21 canastas básicas totales (CBT) mensuales expresadas en equivalente adulto ${ }^{5}$, quedando expuesta la comprometida subsistencia de la familia y capitalización de la unidad de producción. Sumado a esto, en el caso 1, el VAB no alcanzaría para cubrir el salario del peón (178.288 \$/año incluyendo SAC). Por este motivo, toma especial importancia el ingreso obtenido por los representantes de la familia fuera de la explotación, como ocurre con estos productores. En ambos casos, los familiares destinan la totalidad o parte de sus ingresos extra agropecuarios a la explotación. Esta situación es relevante porque si los ingresos logrados fuera de la unidad de producción superan a los obtenidos en el sistema productivo (como en realidad ocurre), el trabajador posiblemente le dedique más tiempo al trabajo no agropecuario corriendo peligro de extinción la unidad de producción familiar.

En relación al endeudamiento, ambos productores mencionaron haber tomado préstamos, específicamente para adquirir herramientas y equipos, insumos y saldar deudas adquiridas previamente. El productor 1 aun transita el periodo de devolución del préstamo, lo que hace aún más complicada su situación en términos económicos.

En ambos casos, toma importancia relevante la búsqueda de alternativas de producción, que permitan incrementar el VBP total.

\section{Estrategia de intervención predial}

En la tabla 6 se observa el VAB producido por una hectárea de $M$. lathyroides para venta de semillas, con un rendimiento promedio de $200 \mathrm{~kg} / \mathrm{h}$.

El CI equivale al gasto en el que se incurre para llevar adelante el proceso productivo. Esto es, contratación de labores de preparación del suelo (rastra de discos), gastos

4.- Se mencionó que el productor 1 contrata un empleado rural permanente, que trabaja 20 horas a la semana en la explotación (el salario rural vigente a marzo de 2020 -sin comida y sin SAC- es de \$30.171,74 \$/mes para peones generales que trabajan 44 horas semanales).

5.- El INDEC (2020) define a la CBT como el valor de los bienes y servicios alimentarios y no alimentarios (vestimenta, transporte, educación, salud, etcétera) que permiten satisfacer mensualmente necesidades consideradas esenciales. En este caso se consideró la CBT expresada por adulto equivalente que, para el mes de marzo de 2020, representó \$13.590,6. Se utilizó la expresión por adulto equivalente porque en ambos casos las familias están compuestas por adultos mayores de edad. Este valor es conocido también como línea de pobreza. 
Tabla 6. Valor agregado bruto producido por una hectárea de M. lathyroides para venta de semillas.

Table 6: Gross value added for the sale of seeds of M. lathyroides (in one hectare of land).

\begin{tabular}{|l|l|}
\hline \multicolumn{2}{|c|}{ PRODUCTO BRUTO } \\
\hline Precio (\$/kg) & 163 \\
\hline Rendimiento (kg/ha) & 200 \\
\hline & \\
\hline & \\
\hline
\end{tabular}

Elaboración propia

\begin{tabular}{|l|c|}
\hline \multicolumn{2}{|c|}{ VALOR AGREGADO BRUTO } \\
\hline Producto Bruto (\$/ha) & 32.698 \\
\hline Consumo intermedio (\$/ha) & 12.522 \\
\hline Valor agregado bruto (\$/ha) & 20.176 \\
\hline Relación VAP/CI (\$/\$) & 1,61 \\
\hline
\end{tabular}

generados por la realización de labores con maquinaria propia ${ }^{6}$ (siembra y corte), gasto en semillas y en cosecha (con la cosechadora propia), sin incluir la mano de obra. La estrategia contempla la implantación de 1 a 5 hectáreas de la leguminosa en cuestión, inicialmente, cuestión que debería reconsiderarse en el caso 1, evaluando la posibilidad de un mayor número de hectáreas dado la necesidad de incrementar el VBP y que posee superficie disponible para ello.

Una sensibilización del VAB realizada en función del rendimiento logrado (entre 100 y 300Kg/ha), sin modificar el consumo intermedio, muestra que este puede variar entre 6.112 \$/ha y 34.239 \$/ha y la relación VAB/CI entre $0,6 \$ / \$$ y $2,3 \$ / \$$.

La incorporación de estas leguminosas a los planteos productivos, aumentaría el VAB total de las explotaciones a través de la venta de las semillas producidas, aunque también debería considerarse un aumento en la productividad del rodeo, generado por los aportes nutricionales de la forrajera (el forraje obtenido en el corte de la legumino- sa que se realiza para mejorar el rendimiento se utiliza como alimento para el rodeo). Este incremento en la productividad de la ganadería no fue evaluado por la falta de información al respecto, pero impactaría de manera positiva en el VA y el VBP de la actividad ganadera.

En ambos casos la implementación de la estrategia aumentaría el valor agregado de la explotación impactando positivamente en el ingreso agropecuario de las familia (una vez descontados los gastos en los que incurren: CI y pago del trabajo asalariado). Este ingreso agropecuario es el que permite cumplir con las obligaciones de las familias propietarias y la sobrevivencia de las explotaciones (e incluso el crecimiento de las mismas). Además de disminuir riesgos propios de este tipo de producciones que se desenvuelven en condiciones de vulnerabilidad (agravadas por el endeudamiento y las condiciones climáticas y de comercialización). En el caso 1, un aumento del ingreso agropecuario es determinante para la sobrevivencia de la unidad de producción

6.- Maquinaria propia compartida entre 5 productores. Si se considerara la maquinaria propia no compartida, el CI ascendería a 14.338 \$/años, el valor agregado bruto sería 18.360 \$/año y la relación VAB/CI sería $1,28 \$ / \$$. 
en el tiempo, lo que demandaría una mejora aun mayor de los resultados de las actividades productivas.

Para llevar adelante esta alternativa, es importante considerar los requerimientos mencionados por Neate y Guei (2011) para el desarrollo exitoso de empresas de semillas de pequeños agricultores: entorno normativo favorable, existencia de una demanda sostenida por semillas de calidad, disponibilidad de variedades mejoradas y fuentes de semillas, iniciativa propia, habilidades técnicas y capacidad, acceso a créditos con bajas tasas de interés, propiedad de empresas y resultados económicos, infraestructura, vínculos entre los sectores formal e informal de semillas, estrategia de comunicación, desarrollo de marcas y marketing.

Si bien este proyecto es innovador para la provincia de Santa Fe, este tipo de propuestas, no es nueva en el mundo. En Tailandia se desarrolla un programa de producción y comercialización de semillas de forrajeras que involucra alrededor de 3.000 pequeños productores (Hare et al, 2013.). Algo similar ocurre en Bolivia, a través de una cooperativa conformada por una Universidad y un grupo de pequeños productores (Sauma y Pizarro, 2007). En el CIAT (Centro Internacional de Agricultura Tropical) se desarrolló y validó una metodología para crear Pequeñas Empresas Semilleras (PES) en campos de pequeños productores (Giraldo Avila, 2005). En Argentina se desarrolla una iniciativa similar entre la Facultad de Ciencias Agrarias de la Universidad Nacional del Nordeste, la Subsecretaría de Agricultura Familiar y un grupo de pequeños productores de la provincia de Chaco (Urbani et al, 2006.).

\section{CONCLUSIONES}

La vulnerabilidad de los sistemas de producción familiares analizados queda expuesta en este trabajo. Los bajos VBP logrados ponen en riesgo su existencia, porque no permiten la retribución del trabajo (familiar o asalariado) y la devolución de préstamos en el caso 1. Esta situación se repite en muchas pequeñas explotaciones familiares. Urge, entonces, la necesidad de evaluar y proponer alternativas mejoradoras que les permitan a los pequeños productores aumentar los ingresos prediales y mitigar los riesgos productivos y de mercados.

El conocimiento de las características particulares de las pequeñas explotaciones familiares ganaderas, a través un análisis técnico y económico por ejemplo, es la base para analizar e implementar cualquier alternativa mejoradora.

En estos dos casos particulares, la propuesta es la incorporación de una leguminosa subtropical, doble propósito, que permitiría incrementar el valor bruto de la producción por la venta de las semillas y por el incremento en la productividad ganadera. La cuestión central de esta propuesta, es el acceso a la maquinaria necesaria, que solo tendrá posibilidades ciertas si se formaliza la comercialización de esta semilla. Los mercados existen, y esto es un motivador inicial interesante. Se debe trabajar, entonces, con los pequeños productores, en cuestiones vinculadas a la práctica del asociativismo en la tenencia de la maquinaria, como alternativa para producción a futuro.

Para desarrollar este tipo de intervenciones, es importante considerar la actitud de los productores frente al riesgo y los objetivos de la familia propietaria de la explotación. Son ellos quienes llevarán adelante 
las actividades y es difícil su continuidad en el tiempo si no las hacen propias. Este tema debería ser abordado en futuras investigaciones.

La información generada en este trabajo, puede servir de base para la formalización de propuestas innovadoras que involucren a una mayor cantidad de productores familiares de características similares a los casos analizados. Esta cuestión debería ser considerada en conjunto entre el sector público y privado, con el único fin de que estas unidades de producción, tan importantes desde el punto de vista de la seguridad alimentaria y de la generación de empleo rural, persistan en el tiempo.

\section{BIBLIOGRAFÍA}

Apollin, F y Eberhart, C. 1999. Análisis y diagnóstico de los sistemas de producción en el medio rural. Guía metodológica. Quito: CAMAREN.

Azcuy Ameghino, E. 2010. Las pequeñas explotaciones de base familiar (Pergamino, 2002): aportes al debate sobre su caracterización y perspectivas. Mundo agrario, 10(20), 00.

Castignani, H. 2011. Zonas Agroeconómicas Homogéneas Santa Fe. Instituto Nacional de Tecnología Agropecuaria. Buenos Aires: INTA.

Fernández, D. 2017. Un análisis sobre distintas estrategias de persistencia de las unidades de producción chacareras en la región pampeana argentina. Agrociencia Uruguay 21(2), pp. 105-115.

Giraldo Avila, G. 2005. Manual para el establecimiento de pequeñas empresas de semillas (PES). Cali, Colombia: Centro Internacional de Agricultura Tropical (CIAT).
Gittinger, J.P. 1983. Análisis económico de proyectos agrícolas. Madrid. España: Ed. Tecnos S.A. Grupo Mercado Común. 2007. Resolución del Grupo Mercado Común (GMC) № 25/07. Directrices para el reconocimiento e identificación de la agricultura familiar en el MERCOSUR. Anexo - III. Montevideo. Uruguay. Disponible en: http://www.sice.oas.org/trade/mrcsrs/resolutions/Res2507.pdf

Hare, M. D., Phengphet, S., Songsiri, T., Sutin, N., Vernon, E. S. y Stern, E. 2013. Impact of tropical forage seed development in villages in Thailand and Laos: Research to village farmer production to seed export. Tropical Grasslands-Forrajes Tropicales, 1(2), pp. 207-211.

INASE. 2016. Catálogo Nacional de Cultivares. Disponible en: https://gestion.inase.gov.ar/consultaGestion/gestiones (Acceso: 12 enero 2020)

Instituto Provincial de Estadística y Censos (IPEC). 2016. Encuesta Ganadera junio 2016. Gobierno de Santa Fe. Disponible en: http:// www.santafe.gov.ar/index.php/web/Estructura-de-Gobierno/Ministerios/Economia/Secretaria-de-Planificacion-y-Politica-Economica/Direccion-Provincial-del-Instituto-Provincial-de-Estadistica-y-Censos-de-la-Provincia-de-Santa-Fe/TEMAS-ESPECIFICOS/Territorio2/Mapas-Dinamicos/ MAPAS-TEMATICOS-DINAMICOS/Mapas-Tematicos-Dinamicos-Sector-Agropecuario (Acceso: 15 noviembre 2019)

INDEC. 2020. Condiciones de vida. Valorización mensual de la canasta básica alimentaria y de la canasta básica total. Gran Buenos Aires. Informes técnicos 4(106). Disponible en: https://www.indec.gob.ar/uploads/informesdeprensa/canasta_06_206B1F43F212. pdf (Acceso: 18 junio 2020)

MAG, IICA. 2014. Caracterización y tipología de la agricultura familiar. San José. Costa Rica: IICA 
Ministerio de Justicia y Derechos Humanos. Sauma, G. y Pizarro, E. 2007. Producción de Presidencia de la Nación. 2014. Ley 27.118. Agricultura familiar. Reparación histórica de la agricultura familiar para la construcción de una nueva ruralidad en la argentina. Disponible en: http://servicios.infoleg.gob.ar/infolegInternet/anexos/240000-244999/241352/ norma.htm (Acceso: 25 enero 2020)

Neate, P.J.H. y Guei, R.G. 2011. Promoción del crecimiento y desarrollo de empresas de semillas de pequeños agricultores en cultivos para la seguridad alimentaria. Mejores prácticas y opciones para la toma de decisiones. Roma, Italia: FAO.

Obschatko, E. 2007. La importancia de la agricultura familiar en la República Argentina. 'En' Barril, G.A y Almada, F. (Eds). La Agricultura Familiar en los países del Cono Sur. Asunción, Paraguay: IICA, pp 7-34.

Reunión Especializada en Agricultura Familiar (REAF). 2016. Estudio de Sistematización de los registros nacionales de la agricultura familiar en el marco de la REAF. Montevideo, Uruguay: Ministerio de Ganadería, Agricultura y Pesca.

Salcedo, S.; De la O, A.P. y Guzmán, L. 2014. El concepto de Agricultura familiar en América Latina y el Caribe. 'En' Salcedo y Guzmán (Eds). Agricultura Familiar en América Latina y el Caribe: Recomendaciones de Política. Santiago, Chile: Organización de las Naciones Unidas para la Alimentación y la Agricultura.

Santangelo, F. y Gil, F. 2016. Potencial productivo de la ganadería bovina de la provincia de Santa Fe. Informe final. Disponible en: https://www.forratec.com.ar/manuales/pdfs/123-20160914123501-pdfEs.pdf (Acceso: 20 diciembre 2019)

semillas forrajeras con pequeños productores en SEFO-SAM, Bolivia. Presentado en XI Seminario "Manejo y Utilización de Pastos y Forrajes en Sistemas de Producción Animal" (pp. 51-59) Venezuela: Universidad Centro Occidental "Lisandro Alvarado" (UCLA) y la Universidad Pedagógica Experimental Libertador (UPEL).

Tommasino, H. y Bruno, Y. 2006. Algunos elementos para la definición de productores familiares, medios y grandes. Disponible en: file:///C:/Users/Usuario/Downloads/tommasino\%20y\%20bruno_.pdf (Acceso: 13 marzo 2020)

Urbani, M. H., Acasuso, C., Marta, L., y Norrmann, G. A. 2006. Tecnología de producción de semilla de "Pasto chané FCA" y su gestión asociada para el desarrollo local en una comunidad agropecuaria del centro-oeste chaqueño. Presentado en Reunión de Comunicaciones Científicas y Tecnológicas 2006 [Resumen A-012]. Argentina: Universidad Nacional del Nordeste.

Zabala, J.M, Pensiero, J., Forni, M., Giavedoni, J., Aiello, F., Yost, A y Quarin, P. 2015. Valorización de los recursos fitogenéticos a través de pequeñas empresas productoras de semillas de forrajeras nativas: evaluación de algunos factores que afectan la producción de semillas en leguminosas forrajeras. Presentado en XVII Foro de Decanos de Facultades de Agronomía del Mercosur, Bolivia y Chile. Red de Cultivos no Tradicionales de Agricultura Familiar. (pp. 65-73). Entre Ríos: Universidad Nacional de Entre Ríos. 